\title{
BMJ Open Paediatric asthma control under a community management model in China: a protocol for a prospective multicentre cohort study
}

Juan Xu, Yong Yin, Hao Zhang, Wen Wei Zhong, Lei Zhang, Jing Zhang, Shu Hua Yuan, Fen Zhang, Li Xia Zhao

To cite: $\mathrm{Xu}$ J, Yin Y, Zhang $\mathrm{H}$, et al. Paediatric asthma control under a community management model in China: a protocol for a prospective multicentre cohort study. BMJ Open 2017;7:e015741. doi:10.1136/ bmjopen-2016-015741

- Prepublication history and additional material is available. To view please visit the journal (http://dx.doi.org/ 10.1136/ bmjopen-2016-015741).

Received 29 December 2016 Revised 19 June 2017 Accepted 21 June 2017
CrossMark

Department of Respiratory Medicine, Shanghai Children's Medical Center affiliated to Shanghai Jiao Tong University of Medicine, Shanghai, China

Correspondence to

Dr Yong Yin;

yinyong9999@163.com

\section{ABSTRACT}

Introduction Childhood asthma is globally one of the most common respiratory disorders and accounts for more school absences and more hospitalizations than any other chronic illness. The worldwide economic burden of this disease exceeds those of HIV/AIDS and tuberculosis combined. Proper intervention and effective management is of paramount importance for the control and prognosis of paediatric asthma. Unfortunately, the rate of uncontrolled and partially controlled paediatric asthma in China is $>90 \%$. This study will use a new management model to investigate the status of asthma control and the adherence of patients to a medication protocol.

Methods This prospective, multicentre, observational study will be conducted at 15 hospitals on children $(\mathrm{n}=800)$ diagnosed with asthma. Each patient will be assigned to either the nearest community hospital or Shanghai Children's Medical Center, whichever is closer to the patient's home, according to the decision of parents. Participants were divided into two groups: tertiary care hospital (Shanghai Children's Medical Center) followup group and community hospital follow-up group. The primary outcome will be the difference in the proportion of controlled, partially controlled and uncontrolled asthma among the two groups. Secondary outcomes will be the differences in adherence rate, lung function, exacerbations, growth development, total asthma-related unscheduled visits, days absent from school and loss of working days for the patient's caregiver. Data will be analysed on an intention-to-treat and a per-protocol basis. Ethics and dissemination Ethics approval was obtained from the Institutional Review Board of Shanghai Children's Medical Center affiliated with Shanghai Jiao Tong University of Medicine. We plan to publish the results of this study in a peer-reviewed journal article.

\section{INTRODUCTION}

Asthma is a common chronic inflammatory disease that can be defined as an airway obstruction that varies over time. ${ }^{12}$ Childhood asthma is globally one of the most common respiratory disorders and accounts for more school absences and more hospitalizations than any other chronic illness. ${ }^{3}$
Strengths and limitations of this study

- This is the first study to unite several hospitals in China to manage paediatric asthma.

- This is the first study to use smartphone applications to collect and download follow-up information.

- Data will be used to objectively evaluate the level of asthma control.

- The study will last for 3 years and requires that a large number of clinicians participate.

There are more than 7.1 million children suffering from asthma in the USA. On average, approximately 1 in 10 children in Canada has asthma. ${ }^{4}$ The healthcare expenditures for asthma are estimated to be more than US $\$ 50.1$ billion per year in the USA, while the loss of productivity of working caregivers has increased to US\$5.9 billion per year. ${ }^{3}$ Worldwide, the economic burden of asthma exceeds that of HIV/AIDS and tuberculosis combined. ${ }^{3}$ In Shanghai, China, the prevalence of asthma in children from 0 to 14 years old was $1.75 \%$ in $1990,4.63 \%$ in 2000 and $7.57 \%$ in 2010, nearly doubling every 10 years. ${ }^{5-7}$ Today, there are more than 20000 asthmatic children in Shanghai. The annual cost of asthma care is estimated to be >US $\$ 400000000$; therefore, paediatric asthma is not just a disease-it is also a societal problem.

Proper intervention and effective management are of paramount importance for the control and prognosis of paediatric asthma. Good asthma control will improve the quality of life, tolerance to exercise and ability to participate in sports and outdoor activities of the sick child. Children with persistent asthma are significantly more likely to continue experiencing asthma symptoms throughout adolescence and adulthood. ${ }^{1}$ In 2013, 80\% of cases of patients with paediatric asthma 
were mildly persistent in China. Unfortunately, the rate of uncontrolled and partially controlled asthma in that country was $>90 \%$. Poorly controlled asthma in children can be very serious and lead to increases in emergency department and other unscheduled visits for asthma symptoms, but the control of paediatric asthma in China is met with many obstacles. Many patients and their caregivers are not very aware of asthma and do not adhere to asthma treatment. In addition, even though there are many asthmatic children, the number of children's hospitals and paediatricians in China is still extremely limited. Nevertheless, the patients are usually taken to a children's hospital to see a doctor, which takes an average of 4-6hours per time and leads to a vicious circle of poor adherence to the prescribed treatment. The reasons for the long visit are that the distance from home to the children's hospital is too long and there are too many people queuing in the children's hospital. It is difficult to quickly increase the number of paediatricians; therefore, it is critical that we establish a new management model for paediatric asthma to improve the current situation.

The objective of this study is to explore a new management model of asthma control in China and to investigate the status of asthma control and the adherence of the patients to prescribed treatment and control methods under this model. We will undergo a 3-year prospective multicentre cohort study to determine whether there is a difference in asthma control rates between asthmatic children who are followed up at Shanghai Children's Medical Center and those followed up at the community hospitals.

\section{METHODS}

Here, we describe the protocol of a prospective, multicentre, observational study. The study will comprise children with asthma attending either Shanghai Children's Medical Center or the other 14 community hospitals in the Pudong District of Shanghai. All patients will be reassessed as to whether they can be diagnosed with asthma according to the asthma guidelines. ${ }^{8}$ Children suspected of having asthma will be given diagnostic treatment but not included in the study until the asthma is confirmed. Once confirmed, we will explain our study to his/her parents as follows: (1) pulmonary physicians of Shanghai Children's Medical Center will go to the community hospital clinics to help in handling asthma cases once every 3 months; (2) all community healthcare providers involved in this clinical study will have received systemic training and all will have the qualifications to diagnose and treat asthma; (3) the treatments of each child with asthma will be based on the guidelines ${ }^{8}$ for treating children with asthma; (4) the 14 community hospitals have the same asthma medication and treatment facilities as Shanghai Children's Medical Center; (5) the distance to the community hospitals is shorter, registration fees are not required and the proportion of medical compensation is higher. It is up to the parents of asthmatic children to follow up at a community hospital or the Shanghai Children's Medical Center. Participants will be divided into two groups as follows: a tertiary care hospital (Shanghai Children's Medical Center) follow-up group and a community hospital follow-up group. For each enrolled patient, basic information and baseline medical conditions will be collected by the investigators using a study-specific application programme (APP). The patients will be assigned to a community hospital or to Shanghai Children's Medical Center, whichever is nearer to the subject's home, for their future onsite visits (V2-V5) and will return to Shanghai Children's Medical Center for their last visit (V6). During the study, patients will receive 12 months of standard treatment prescribed by the investigators according to 'Guidelines for the Diagnosis and Optimal Management of Asthma in Children. ${ }^{8}$ An asthma control questionnaire and test will be completed by the patients' caregivers each month. Global outcomes will be quantified using the test scores, lung function, adherence rate and exacerbation data collected during the observation period.

\section{Sponsorship}

This is a clinician-initiated study funded by the Science and Technology Commission of Shanghai Municipality and by Shanghai Shen-kang Hospital Development Center. It is hosted by Shanghai Children's Medical Center. Both the Shanghai Committee of Science and Technology and Shanghai Shen-kang Hospital Development Center are government-funded institutions that focus on the development of new scientific and technological protocols. The trial was approved by the Shanghai Children's Medical Center ethics committee and is registered as NCT02649803 (https://clinicaltrials. gov). A detailed statistical plan has been approved by an independent statistical team. The study-specific APP was created and supplied by Shanghai Yitong Information Technology Corporation LTD.

\section{Setting}

This is a prospective, multicentre, observational study of two management strategies to learn more about paediatric asthma control. The study is being conducted at the Shanghai Children's Medical Center and 14 community hospitals. All community healthcare providers involved in this clinical study received systemic training on asthma diagnosis and treatment, and all were eligible to participate. The study-specific APP, called 'asthma relief,' comprises follow-up assessments, weather alerts, health guidance and the asthma community. The study period is from 2016 with ongoing recruitment.

\section{Participants}

Before recruitment, an asthma diagnosis will be made by a clinician. The patient who fulfils criteria $1-4$ or criterion 4 plus any item of criterion 5 could be diagnosed with paediatric asthma. The criteria are as follows: (1) recurrent respiratory symptoms (wheeze, cough, dyspnoea, chest tightness); typically worse at night/early morning; exacerbated by exercise, viral infection, smoke, dust, pets, mould, dampness, weather changes, laughing, crying or 
allergens; (2) a high-pitched whistling sound, usually during exhalation, detected in both sides of the lung by auscultation of the chest; (3) symptoms/signs could be relieved automatically or by antiasthmatic treatment; (4) no other diseases that might also cause wheeze, cough, dyspnoea or chest tightness; (5) atypical symptoms/signs without wheeze or whistling sound but fulfilling at least one of the following (a) positive results of bronchial provocation or exercise challenge tests; (b) reversible airflow limitations as follows: (i) the result of bronchial dilation test is positive; (ii) antiasthmatic treatment is effective for the patient; (c) the ratio of daily variation of peak expiratory flow (PEF) is $>20 \%$.

\section{Inclusion criteria}

Patients will be eligible to participate if all the following criteria apply: (1) male or female outpatient $<18$ years old, (2) diagnosed with asthma according to the Guidelines for the Diagnosis and Optimal Management of Asthma in Children, ${ }^{8}$ (3) consent obtained by subject's parent or guardian and (4) subjects or subject's caregiver has a smartphone at their disposal.

\section{Exclusion criteria}

Patients will not be eligible to participate if any of the following exclusion criteria are present: (1) differential diagnosis of asthma such as congenital heart disease, gastro-oesophageal reflux, bronchopulmonary dysplasia or bronchiolitis obliterans; (2) allergy to any inhaler cortical steroid; (3) other diseases that could interfere with the study results judged by the clinicians; (4) participation in any analogous clinical study within 3 months.

\section{Study design}

The study design flow is shown in figure 1. All community healthcare providers involved in this clinical study will receive systemic training before the initial patient enrolment. Only qualified providers can participate in this study. Patients identified at Shanghai Children's Medical Center and the 14 community hospitals who meet the inclusion, and not the exclusion, criteria are invited to participate in the study. Once consented, a signed informed consent form will be submitted and each patient visit will be overseen by a registered clinician.

At the initial visit (V1), the participant's sex, age, body weight, height, medical/surgical history, control status assessment, exacerbation assessment, asthma medication (if applicable), inhalation device information (if applicable) and concomitant medications will be recorded. The Childhood Asthma Control Test (c-ACT) score and Asthma Quality of Life Questionnaire ${ }^{9}$ will be completed by the patient's caregiver. The patients $>5$ years old will receive PEF and Fraction of Exhaled Nitric Oxide (FeNO) tests for evaluation of lung function and inflammation level.

The patient's caregiver will be instructed to install the APP on their smartphone and learn how to use it. The patients will be assigned to the nearest community hospital or Shanghai Children's Medical Center, whichever is nearer to their home, for their future onsite visits. Asthma medicine will be prescribed by the clinician according to the patient's asthma severity and control status in line with the Guidelines for the Diagnosis and Optimal Management of Asthma in Children. ${ }^{8}$

The patient's caregiver will be prompted to fill out a monthly asthma control questionnaire using the smartphone APP. This questionnaire contains questions regarding prescription renewals, adherence to prescribed treatment protocols, control status, asthma attacks and health-related economic information. Health-related economic information includes days the patient was absent from school, days the caregiver could not go to work and total cost of the hospital visits, including the registry fee and costs for medication, hospitalisation and travel. The caregiver will also be asked to fill out an asthma control test for patients $\geq 12$ years old (online supplementary appendix A), c-ACT for patients 5-12 years old (online supplementary appendix B) or Global Initiative for Asthma (GINA) assessment of asthma control in children $\leq 5$ years old (online supplementary appendix $\mathrm{C}$ ).

Patients will be requested to visit prearranged community hospitals at months 1, 3, 6 and 9 (V2-V5) and arrange their last visit with Shanghai Children's Medical Center at month 12 (V6). At each visit, control status assessment, exacerbation assessment, asthma medication, inhalation device information, concomitant medications and health-related economic information will be collected by the clinicians. Patients will receive PEF ( $>5$ years old) and $\mathrm{FeNO}$ tests for evaluation of lung function and inflammation level. After comprehensive assessment of the patient's symptom control, risk factors, occurrence of exacerbations and practical issues (cost, ability to use the device and adherence to treatment protocols), the clinician will decide whether to step up or step down the asthma treatment.

\section{Data collection}

Asthma control questionnaire and asthma control test score

The asthma relief APP compromises follow-up assessments, weather alerts, health guidance and the asthma

\begin{tabular}{|c|c|c|c|c|c|c|c|c|c|c|c|c|}
\hline \multicolumn{2}{|c|}{$\begin{array}{l}\text { Enrolment visit (V1) at } \\
\text { Shanghai Children's Medical } \\
\text { Center }\end{array}$} & \multicolumn{9}{|c|}{$\begin{array}{l}\text { Visists 2-5 (V2-V5) at the nearest co-operation community hospital or Shanghai } \\
\text { Children's Medical Center, whichever is nearer to the patient's home (pre-assigned } \\
\text { during V1) }\end{array}$} & \multicolumn{2}{|c|}{$\begin{array}{c}\text { Final visit (V6) at } \\
\text { Shanghai Children's } \\
\text { Medical Center }\end{array}$} \\
\hline \multicolumn{2}{|l|}{ Onsite visits } & & & & & & & & & & & \\
\hline Month & 1 & 2 & 3 & 4 & 5 & 6 & 7 & 8 & 9 & 10 & 11 & 12 \\
\hline Questionnaires & & & & & & & & & & & & \\
\hline
\end{tabular}

Figure 1 The study design flow. 
community. Follow-up assessment will be determined by the asthma diary, asthma control questionnaire, c-ACT, Paediatric Asthma Quality of Life Questionnaire and growth development assessment. APP will be available for free downloading from a mobile phone market. The asthma diary will be updated by the patient's caregiver each day. The asthma control questionnaire, c-ACT and growth development assessment will be completed by the patient's caregiver each month using APP. All data stored in the APP will be wirelessly uploaded into an MS Excel database through an internet server. No changes can be made to the database after the data are uploaded.

\section{Lung function}

Participants will receive a lung function test at each visit, the results of which are then collected at the subsequent visit. We will equip the 14 community hospitals with simple pulmonary instruments.

\section{Objectives}

The objective of this study is to assess whether the asthma control status of the patients under community hospital management is as well as that for the patients under Shanghai Children's Medical Center.

\section{Primary outcome}

The overall proportion (per patient per visit) of controlled, partially controlled and uncontrolled asthma between the two groups (patients recruited into the 14 community hospitals and patients assigned to Shanghai Children's Medical Center) will be compared.

For patients $\geq 12$ years old, c-ACT will be used to determine their asthma control status. Scores range from 5 to 25 , with higher scores indicating better results. Scores of 20-25 are classified as well-controlled asthma, 16-20 as partially controlled asthma and 5-15 as uncontrolled asthma. For patients 6-11 years old, c-ACT will be used to determine their asthma control status. Scores range from 0 to 27 , with higher scores indicating better results. Scores of 23-27 are classified as well-controlled asthma, 20-22 as partially controlled asthma and 0-19 as uncontrolled asthma. For patients $\leq 5$ years old, the GINA assessment will be used to determine their asthma control status. The GINA assessment comprises four questions answered either 'yes' or 'no.' If all answers are no, the conditions will be classified as well-controlled asthma; if there are one or two yes answers, the conditions will be classified as partially controlled asthma; if there are three to four yes answers, the condition will be classified as uncontrolled asthma.

\section{Secondary outcomes}

The rate of adherence, lung function, exacerbations, growth development, total times (per patient) for asthma-related unscheduled visits, total days absent from school from asthma conditions and the total loss of working days for the patient's caregiver from asthma conditions between the two groups will be compared at the end of the study.
The total amount of asthma drugs consumed between each visit and the total amount of asthma drugs prescribed between two consecutive visits will be recorded. When the total amount of asthma drugs consumed exceeds the total amount of asthma drugs prescribed, the total amount of asthma drugs prescribed will be used for the calculation of the cumulative amount of asthma drugs consumed. For each patient, adherence will be calculated as the cumulative amount of asthma drugs consumed during 12 months divided by the cumulative amount of asthma drugs prescribed during 12 months. Adherence calculated in this way is a continuous variable ranging from $0 \%$ to $100 \%$ (censored at $100 \%$ ), which will then be dichotomized as good $(>80 \%)$ and poor $(<80 \%)$ adherence. The cut-off value of $80 \%$ is determined according to previous studies that showed that adherence $>80 \%$ is associated with good asthma control status. ${ }^{11}{ }^{11}$ Exacerbations are defined as an increase in symptoms (ie, shortness of breath, wheeze, cough) that would require a course of systemic glucocorticoids. The growth development information will be collected using APP. The total asthma-related unscheduled visits will be defined as hospitalisation resulting from an asthma attack, emergency visits resulting from an asthma attack or oral corticosteroids prescribed at a clinic because of an asthma attack. The total cost of all asthma-related hospital visits will include the registry fee and the costs for medication, hospitalisation and travel.

\section{Sample size calculation}

The sample size will be estimated to be 800 subjects. We performed a pretest study on the complete control rate of asthma in both Shanghai Children's Medical Center and two community hospitals of Shanghai. The results were $35.2 \%$ and $25.7 \%$, respectively. Our hypothesis is that there is a significant difference in asthma control rates between asthmatic children who are followed up at the tertiary care hospital and those followed up at the community hospitals. In community hospital follow-up group, it is assumed that the rate of asthma control is $25.7 \%$; thus, there would be sufficient power to detect an increased rate of at least $10 \%$ in tertiary care hospital (Shanghai Children's Medical Center) follow-up group, using the planned sample size. Figure 2 shows the formulas used to computer sample size.

The sample size will be 400 subjects in each group; loss to follow-up is estimated at $10 \%$, which will allow the results to show a $10 \%$ difference $(0.8$ power) in asthma control status between group proportions with a type I error probability of 0.05 .

\section{Statistical methods}

Epidemiological methods will be used for data analysis. Statistical analysis will be performed using SAS; the version used will be specified in the Statistical Analysis Plan (SAP). A comprehensive SAP will be prepared before database lock. Data will be analysed on an intention-to-treat and a per-protocol basis. Significance will be set at 5.0\%. Descriptive statistics will be used for all the data analyses as follows: categorical variables by frequency 
$n A=\kappa n B$

$\kappa=\frac{n A}{n B}$

$n B=\left(\frac{p A(1-p A)}{\kappa}+p B(1-p B)\right)\left(\frac{z(1-\alpha / 2)+Z(1-\beta)}{p A-p B}\right)^{2}$

$1-\beta=\Phi(Z-Z(1-\alpha / 2))+\Phi(-Z-Z(1-\alpha / 2))$

$Z=\frac{p A-p B}{\sqrt{\frac{p A(1-p A)}{n A}+\frac{p B(1-p B)}{n B}}}$

Figure 2 Formulas for sample size calculations. $k=n A / n B$ is the matching ratio. $\Phi$ is the standard normal distribution function. $\Phi^{-1}$ is the standard normal quantile function. $\alpha$ is type I error. $\beta$ is type II error, meaning $1-\beta$ is power. $p A=0.352, p B=0.257, \alpha=0.05, \beta=0.2, \kappa=1$.

tables (ie, number of evaluable subjects, frequency and percentage for categorical values) and continuous variables by sample statistics (ie, mean, SD, minimum, median and maximum). More details will be described in SAP. Categorical variables will include the proportion of patients with controlled, partially controlled and uncontrolled asthma, as well as the percentage of patients with good adherence; continuous variables will include the total times for asthma-related unscheduled visits, total cost of all asthma-related hospital visits, total days absent from school because of asthma conditions and total loss of working days for patient's caregiver because of asthma conditions.

\section{DISCUSSION}

According to the newest report on paediatric resources in China by Sunkun, chair of the Chinese Academy of Paediatrics, there are 86042 paediatrician deficiency in China. In addition, the imbalance between paediatric and adults resources in China is prominent. Concurrently, the prevalence of asthma in children from 0 to 14 years old was $7.57 \%$ in $2010,{ }^{5-7}$ and there are $>20000$ children with asthma in Shanghai. Asthma is a type of self-management disease. Research results by Morton et $a l^{10}$ showed that the average compliance with asthma treatment was $87.0 \%$ among children aged 2-6 years. The study of Wardenier et $a l^{12}$ also indicated that adherence to childhood asthma treatment was as high as $76.5 \%$; however, Vasbinder et al ${ }^{13}$ found that adherence was only $49.3 \%$. Another study showed that the rate of compliance rate with asthma treatment decreased from $60.4 \%$ to $49.8 \%$ within 8 months after the initial visit and diagnosis. ${ }^{14}$ Meanwhile, Shen et $a l^{15}$ discovered that the adherence rate was only $32.5 \%$, and the proportion of patients who never come back to hospital was as high as $46.2 \%$. Jentzsch et $a l^{14}$ also found that poor compliance was an independent risk factor of poor asthma control; therefore, it is necessary to establish a new management model for paediatric asthma in China.

In recent years, economic development in China along with the development of more medical resources in its community hospitals has increased. The quality of healthcare in the community hospitals could well meet the requirements for the control of paediatric asthma, and these hospitals have enough resources to provide patients with treatment and education. In addition, it is more convenient and economical for caregivers to make the scheduled doctor visits in a community hospital that is near their homes. We believe that it is feasible to explore a new and effective asthma management model at the community hospitals.

Adherence to prescribed treatment protocols depends mainly on the caregivers to help children with asthma take their medication; however, many caregivers are not educated on the importance of taking the medication regularly, and most are worried about side effects ${ }^{16}$; therefore, we will hold monthly lectures on asthma for caregivers in the local kindergartens and primary schools.

With the proliferation of smartphone devices in big cities such as Shanghai, it is practical to use these devices in this study to collect data from our patients or their caregivers. Data collection using a smartphone can improve data quality and increase efficiency at both the data recording and entry stage, which could lead to substantial time and cost savings for both the patients and the physicians. It is expected that some caregivers will forget to complete the follow-up information in the APP over the study period; therefore, all of caregivers will be added to a specific WeChat group when they sign the informed consent, and we will arrange for two research assistants to remind each of them through WeChat. In addition, some caregivers might not be able to understand or master APP, in which case they will be provided with a paper questionnaire.

This study has several new features. It is the first study to unite community hospitals with the children's hospital to manage paediatric asthma in China. It is the first study to use a smartphone application to collect follow-up information, which can also automatically and synchronously upload to a database. In addition, by collecting the data on lung function, c-ACT, GINA assessment of asthma control and clinical outcomes, we will be able to objectively evaluate the level of asthma control; however, the limitations are that the study will last for 3 years and will require that a large number of clinicians participate.

With regard to safety, all community healthcare providers involved in this study will receive systemic training before initial patient enrolment. The training will comprise two parts. In part I, trainees will receive two lectures on the basic knowledge and management strategies of paediatric asthma and the protocols on implementing this clinical study. After each lecture, the trainees will be required to pass a quiz with a score of $85 \%$. In part II, each community physician and clinician involved in this study will be required to learn standard 
clinical practice at Shanghai Children's Medical Center once a week and once every 2 weeks, respectively. The training process will last 2 months, during which community physicians and clinicians will be trained at both the clinics and wards of Shanghai Children's Medical Center. They will also learn how to correctly operate spirometers and atomising pumps. After training, each trainee will be scored by their mentor according to their clinical performance. Only qualified trainees will be able to participate in the study.

Patients can withdraw at any time during the study without any impact on their clinical care. In addition, if the clinician believes there is a clinical indication to do so, a patient can be removed from the study in his/her best interest. This is a prospective study with non-interventional character and no specific drug. No proactive safety data will be collected; however, according to China regulations (ie, Provisions for Adverse Drug Reaction (ADR) Reporting and Monitoring, Order No. 81 of the Ministry of Health), the clinicians should report any ADR identified during their assessment of the questionnaire data.

In summary, this study is proposed to explore a new and effective management model to monitor paediatric asthma under community hospitals in China.

\section{ETHICS AND DISSEMINATION}

Ethics approval was obtained from the institutional review board of Shanghai Children's Medical Center affiliated with Shanghai Jiao Tong University of Medicine. We plan to publish the results of this study in a peer-reviewed journal article. We will report between-group differences for all outcomes. The study results will also be disseminated through presentations at national and international conferences.

Acknowledgements The authors thank Shanghai Yitong Information Technology Corporation LTD for its technological support.

Collaborators Sheng Chen and Lihua Qin, Beicai Community Health Service Center, Shanghai, China. Ying He and Yiqin Cai, Heqing Community Health Service Center, Shanghai, China. Qunzhi Wu, Lianyang Community Health Service Center, Shanghai, China. Huihua Qiao and Xiaogin Liu, Hudong Community Health Service Center, Shanghai, China. Binbin Zhang and Dandan Mo, Jinyang Community Health Service Center, Shanghai, China. Lanping Ye, Zhuqiao Community Health Service Center, Shanghai, China. Lingjun Liu and Yumin Zhang, Lujiazui Community Health Service Center, Shanghai, China. Qinqin Lu and Yan Yin, Gaoqiao Community Health Service Center, Shanghai, China. Hui Dai, Sanlin Community Health Service Center, Shanghai, China. Lihua Qu, Datuan Community Health Service Center, Shanghai, China. Yichun Yuan, Yangjing Community Health Service Center, Shanghai, China. Zhigang Zhou, Tangqiao Community Health Service Center, Shanghai, China. Wei Feng, Huinan Community Health Service Center, Shanghai, China. Yanping Wang, Nicheng Community Health Service Center, Shanghai, China.

Contributors JX contributed to the drafting of the manuscript. YY conceived of and designed the study. HZ, WWZ, LZ and JZ made substantial contributions to study design. SHY provided statistical support. FZ, SHY and LXZ were responsible for collecting data. All authors will be involved in writing the manuscript and revising it critically for intellectual content, and all will give final approval of the version to be published.

Funding This project was funded by the Science and Technology Commission of Shanghai Municipality (No. SHDC12016216) and Shanghai Shen-kang Hospital
Development Center Projects for the Prevention and Control of Chronic Diseases (No. SHDC12015306). It was also funded by the Key Projects of Shanghai Science and Technology Department of Medicine (16411955100) and by the Three Years ActionPlan to Promote Clinical Skills and Clinical Innovation Capability in Municipal Hospitals (16CR4002A).

Competing interests None declared.

Patient consent Parental/guardian consent obtained.

Ethics approval Institutional Review Board of Shanghai Children's Medical Center affiliated to Shanghai Jiao Tong University of Medicine.

Provenance and peer review Not commissioned; externally peer reviewed.

Open Access This is an Open Access article distributed in accordance with the Creative Commons Attribution Non Commercial (CC BY-NC 4.0) license, which permits others to distribute, remix, adapt, build upon this work non-commercially, and license their derivative works on different terms, provided the original work is properly cited and the use is non-commercial. See: http://creativecommons.org/ licenses/by-nc/4.0/

(c) Article author(s) (or their employer(s) unless otherwise stated in the text of the article) 2017. All rights reserved. No commercial use is permitted unless otherwise expressly granted.

\section{REFERENCES}

1. Kovesi T, Giles BL, Pasterkamp H, et al. Long-term management of asthma in first nations and Inuit children: a knowledge translation tool based on canadian paediatric asthma guidelines, intended for use by front-line health care professionals working in isolated communities. Paediatr Child Health 2012;17:46-64.

2. Lougheed MD, Lemière C, Dell SD, et al. Canadian Thoracic Society Asthma Management Continuum-2010 Consensus Summary for children six years of age and over, and adults. Can Respir $J$ 2010;17:15-24.

3. Noutsios GT, Floros J. Childhood asthma: causes, risks, and protective factors; a role of innate immunity. Swiss Med Wkly 2014;144:w14036.

4. Kovesi T, Schuh S, Spier S, et al. Achieving control of asthma in preschoolers. CMAJ 2010;182:E172-E183.

5. The National Cooperative Research Group on Childhood Asthma. A nationwide survey on the state of asthma in a population of 900 0-14-year-old children. Chin J Tuberc Respir Dis 1993;16:64-8.

6. The National Cooperative Group on Childhood Asthma. A nationwide survey in China on prevalence of asthma in urban children. Chin J Pediatr 2003;41:123-7.

7. The National Cooperative Group on Childhood Asthma, Institute of Environmental Health and Related Products. Third nationwide survey of childhood asthma in urban areas of China. Chin J Pediatr 2013;51:729-35.

8. The Subspecialty Group of Respiratory Diseases, The Society of Pediatrics. Chinese Medical Association, The Editorial Board, Chinese Journal of Pediatrics. Guideline for the diagnosis and optimal management of asthma in children Chin $\mathrm{J}$ Pediatr 2008;46:745-53.

9. Juniper EF, Guyatt GH, Epstein RS, et al. Evaluation of impairment of health related quality of life in asthma: development of a questionnaire for use in clinical trials. Thorax 1992;47:76-83.

10. Morton RW, Everard ML, Elphick HE. Adherence in childhood asthma: the elephant in the room. Arch Dis Child 2014;99:949-53.

11. Klok T, Kaptein AA, Duiverman EJ, et al. It's the adherence, stupid (that determines asthma control in preschool children)! . Eur Respir J 2014;43:783-91.

12. Wardenier NR, Klok T, de Groot EP, et al. Height growth in children with asthma treated with guideline-recommended dosages of fluticasone and electronically assessed adherence. Arch Dis Child 2016;101:637-9.

13. Vasbinder E, Dahhan N, Wolf B, et al. The association of ethnicity with electronically measured adherence to inhaled corticosteroids in children. Eur J Clin Pharmacol 2013;69:683-90.

14. Jentzsch NS, Camargos P, Sarinho ES, et al. Adherence rate to beclomethasone dipropionate and the level of asthma control. Respir Med 2012;106:338-43.

15. Shen KL, Zhang GQ, Zhao J, et al. Questionnaire of the asthma children's parents in Beijing. Chin J Pediatr 2009;47:942-5.

16. Desai M, Oppenheimer JJ. Medication adherence in the asthmatic child and adolescent. Curr Allergy Asthma Rep 2011;11:454-64. 